\title{
Review of Dejian Mind-Body Intervention Used to Children with Autism Spectrum Disorder
}

\author{
Ming-Yuan Zhao ${ }^{1}$, Chen $\mathrm{Li}^{1}$, Na Zhao ${ }^{2}$ and Si-Min Huang ${ }^{1 *}$ \\ ${ }^{1}$ College of Physical Education and Health, Zhaoqing University, Guangdong Zhaoqing 526061, China \\ ${ }^{2}$ School of clinical medicine , Qiqihaer medical university, Qiqihaer, 11230, China \\ *Corresponding author: Si-Min Huang (e-mail: 490819896@qq.com)
}

\begin{abstract}
The purpose of this study is to summarize research investigating the potential benefits of the Dejian Mind-Body Intervention (DMBI) in enhancing the functioning of children with autism spectrum disorder (ASD) as well as further explore its potential mechanisms. The DMBI is the organic integration of Shaolin Chanwuyi and modern psychosomatic medicine. It is unique and has substantial effects on children with ASD. The DMBI primarily relies on traditional health regimens such as Neiyanggong (Qigong of the Shaolin Temple), Zen meditation (meditation of the Shaolin Temple), regulated diet, and Tongqiao (keep bodily opening healthy) to enhance patients' brain functioning, thereby improving self-control, promoting mental development, and reducing repetitive behaviors. The DMBI is safe, effective, and economical, making it worthy of wider attention. The DMBI is recognized as possessing a distinct advantage in mitigating the symptoms of ASD in children. Hence, it has potential to serve as an intervention in rehabilitation and medical institutions that specialize in the treatment of children with ASD.
\end{abstract}

Keywords: Dejian Mind-Body Intervention, children with autism spectrum disorder, Chinese Chan practice

\section{INTRODUCTION}

Autism spectrum disorder (ASD) refers to a complex neurological and developmental disorder that encompasses isolated communication impairment, social impairment, and repetitive patterns of behavior [1]. Previous studies have showed that such symptoms usually emerge in early stages of child development. ASD is considered a severe mental disorder in children that hinders normal development and seriously impairs activities of daily living and quality of life. [2]. Furthermore, the secondary problems caused by these obstacles even exceed the ones themselves. Firstly, ASD incurs severe psychological and financial burdens on families, who must manage multiple issues [3]. Secondly, evidence has suggested that the rates of suicidality increase almost three and five times in ASD community and inpatient samples compared with a control group [4]. Lastly, it is difficult for children with ASD to completely socially integrate, for their symptoms influence communication and socialization throughout the life span [5].

In China, problems surrounding children with ASD are becoming more serious every year [6]. According to the latest "Report on the Development of China's Autism Education and Rehabilitation Industry III" (2019), the incidence of autism in China is $1 \%$, and there are currently more than 13 million people with ASD, 3 million of which are 12 years old or younger [7]. Moreover, in addition to the fact that the number of children with ASD has increased, there is a high proportion that overlaps with attention-deficit hyperactivity disorder (ADHD) in child patients Specifically, $20 \%-50 \%$ of children clinically diagnosed with ADHD also fit the diagnostic criteria for ASD, and $30 \%-80 \%$ of children diagnosed with ASD fit the diagnostic criteria for ADHD [8]. Thus, the choice of intervention for children with ASD is critical.

Nevertheless, the present treatment for children with ASD chiefly employs training interventions and medication. The research and administration of these treatments are largely based on Western medical knowledge and philosophy, including Treatment and Education of Autistic and Communication Handicapped Children (TEACCH) [9], Sensory Integration Training (SIT) [10], Applied Behavior Analysis (ABA) [11], Relationship Development Intervention (RDI) [12], and 
Dance Movement Therapy (DMT) [13]. Whether health regimens originating from traditional Chinese culture have any effect on children with ASD is unknown. Furthermore, if modern medical value can be found in traditional Chinese culture, then the mechanisms that facilitate improvements in a patient' $s$ condition may also be a topic worthy of investigation.

For the above mentioned problems, especially regarding the principle of improving patients' brain functions to alleviate their symptoms, Professor Agnes S. Chan and her team from the Department of Psychology at the Chinese University of Hong Kong have explored the potential benefits of the DMBI on the basis of traditional regimens. Studies have shown favorable results in children with ASD after participating in the intervention [14]. In this study, the progress in related research on the DMBI in treatment of ASD is summarized, and potential mechanisms are further discussed.

\section{OVERVIEW OF THE DEJIAN MIND- BODY INTERVENTION}

The DMBI is a Chinese Chan-based lifestyle intervention that originated from Chanwuyi tradition (i.e., Zen, martial arts, and healing) and was developed based on the traditional Zen martial art and Zen medicine from the Shaolin Temple [15]. Specifically, it embodies the essence of Chanwuyi theories and practices passed down in the Yonghuatang (the Nanyuan, or Southern Courtyard) headed by a grand master named Shi Dejian (a Shaolin monk), an inheritor of Chanwuyi. The DMBI was developed and named in honor of Shi Dejian by Professor Chan, who integrated Chanwuyi with modern Western brain science, forming a wholesome mind-body intervention. In particular, the DMBI is based on the Neiyanggong, a health exercise derived from Shaolin Qigong. It incorporates Zen meditation, a regulated diet, and Tongqiao (keep bodily opening healthy), making it a convenient, easy-to-learn, and highly effective intervention, as shown in Table 1. Through a series of mind-body exercises, the DMBI aims to improve physical and psychological health.

TABLE 1 Main contents of the DMBI

\begin{tabular}{ll}
\hline Content & \multicolumn{1}{c}{ Key Points } \\
\hline Xu zhuang (tranquil standing), natural Dan Tian \\
breathing, self-initiated Dan Tian breathing, songjianshi \\
(shoulder relief exercise), moyushi ("catching a fish" \\
exercise), fengbailiu ("willow in the wind" exercise), \\
mubifa (nasal bridge massage), and shougong (closing \\
moves). \\
Practicing Zen meditation to improve one's \\
temperament, morality, and compassion. \\
Alight vegetarian diet that avoids any food items that are \\
Regulated diet & strong in smell, made of flesh, spicy, or contain eggs. \\
Tongqiao & Freeing of clean orifices, freeing of unclean orifices. \\
\hline
\end{tabular}

The DMBI pursues physical and psychological harmony and renounces practices that only focus on physical symptoms and do not improve the quality of life for patients and their families. Aside from benefitting physical health, it promotes the improvement of mental, ideological, and moral qualities through the development of positive attitudes and behaviors. In this regard, the DMBI is an amalgamation of traditional and relatively unorthodox modern-day psychological, life science, and medical philosophies.

\section{PATHWAYS AND PHYSIOLOGICAL MECHANISMS THROUGH WHICH THE DMBI AFFECTS CHILDREN WITH ASD}

\subsection{PATHWAYS THROUGH WHICH THE DMBI AFFECTS CHILDREN WITH ASD}

\subsubsection{Neiyanggong}

Neiyanggong is a model of mental exercise and is the most important part of DMBI, which was developed by Chanwuyi inheritor Shi Dejian through the combination of ancient Shaolin martial arts and modern health philosophies [16]. Neiyanggong consists of eight gentle and calm movements and requires approximately 30 minutes to reduce stress, enhance strength, and flexibility in all four limbs [17].

In addition to the abovementioned characteristics, rhythmic breathing combined with slow movements are the main features of Neiyanggong. That is, Neiyanggong is a special mind-body exercise. In this way, regular practice of Neiyanggong can gradually improve selfcontrol problems and sensory over-responsivity (SOR) [18], which are common and manifest in children with ASD. Regular practice can also improve overall physical health and the circulation of $Q i$ in the blood.

\subsubsection{Zen meditation}

Zen meditation is a type of Buddhist mind-body exercise methods in ancient China [19]. On the basis of mind-body exercise methods, Zen meditation in the DMBI is markedly different from Zen meditation in the aforementioned well-known therapies. DMBI Zen meditation does not involve purely static meditation. Instead, it seeks active integration of Zen philosophies in daily life and enlightenment from trivial details in everyday activities.

Zen meditation in the DMBI can benefit children with ASD by reducing stress and changing lifestyle through breathing and mindfulness. Thus, Zen meditation in the DMBI in daily life is key to learning self-awareness and maintaining mental relaxation. 


\subsubsection{Vegetarian diet}

The DMBI system involves a special vegetarian diet, which is especially rigorous (shown in Table 2) and places special emphasis on changing the dietary habits of patients during the intervention. The philosophy of the DMBI vegetarian diet argues that treating a disease solely by pharmaceutical or therapeutic means without rectifying dietary habits is futile, because it merely alleviates the symptoms and does not cure the disease.

Diet is a frequently used intervention in children with ASD, for research has shown abnormalities in gastrointestinal symptoms, a higher proportion of which may be associated with the human gut microbiota [20]. Thus, a therapy targeting the condition of intestinal microbes is feasible.

Furthermore, several recent studies have supported the benefits of a regulated diet, which are particularly notable in children with ASD, whose micronutrient conditions suggest that they have a generally low intake of vegetables [21, 22].

TABLE 2 Vegetarian diet regimen of the DBMI

\begin{tabular}{cccc} 
Food & $\begin{array}{c}\text { Western } \\
\text { vegetarian diet }\end{array}$ & $\begin{array}{c}\text { Buddhist } \\
\text { vegetarian diet }\end{array}$ & $\begin{array}{c}\text { DBMl vegetarian } \\
\text { diet }\end{array}$ \\
Feat & No & No & No \\
Eggs & Yes & No & No \\
Spring onion & Yes & No & No \\
Garlic & Yes & No & No \\
Ginger & Yes & Nos & No \\
Mustard & Yes & Yes & No \\
Spice & Yes & Yes & No \\
Pepper & Yes & Yes & No \\
Rape & Yes & Yes & No \\
Coriander & Yes & Yes & No \\
\hline
\end{tabular}

\subsubsection{Tongqiao}

Tongqiao refers to keeping all body orifices (i.e., nose, ears, and intestines) free from obstructions. According to the principle of the DMBI, the blockage of orifices (i.e., bodily openings) is the root of many mental and physical health problems [23]. Therefore, Tongqiao is an integral and unique feature of the DMBI. It is a healthcare and treatment concept that has not yet been practiced in Western medicine.

Specifically, Tongqiao is a comprehensive treatment. It includes not only physical massage methods (i.e., massaging the nose repeatedly) but also Chinese herbal medicine methods. Practice of Tongqiao is good for improving brain function in children with ASD.

\subsection{PHYSIOLOGICAL MECHANISMS THROUGH WHICH THE DMBI AFFECTS CHILDREN WITH ASD}

To date, physiological mechanisms and causes of ASD are not entirely clear. Some studies have proposed that the dysfunction of cerebral tissues may be a major cause because it is significantly correlated with the incidence of ASD. To clarify, cerebral perfusion deficits in the temporal lobe [24] have been discovered as a potential cause of abnormal cognitive [25], social-behavioral [26], and linguistic [27] functions of relevant cerebral tissues. In particular, in individuals with ASD, frontal lobe hypofunction has been identified as a cause of decreased top-down executive control and cognitive activity, which can in turn cause repetitive behaviors [28]. Additionally, studies have revealed that brain-derived neurotrophic factor may be related to ASD [29] and that changes in the maternal gut microbiome may increase the risk of ASD in children [30].

Several studies have reported that children with ASD may differ from neurotypical children in terms of cerebral structure and functioning. One example is a study by Chan et al., who used the DMBI as an intervention for improving dietary habits, daily life patterns, and mental statuses of children with ASD. After the intervention was administered, they recorded participants' electroencephalogram (EEG) activity and employed functional magnetic resonance imaging (fMRI) to collect brain images corresponding to different intervention programs. Moreover, they used various scales to assess behavioral, cognitive, and emotional dimensions.

Notably, even though the DMBI is composed of elements that are closely associated with each other, Chan et al. were able to achieve more than applying the DMBI as a complete set. They investigated the effectiveness of individual elements of the DMBI (i.e., Neiyanggong, Tongqiao, Dan Tian breathing [DTB] methods, and regulated diet) in children with ASD.

\subsubsection{Effects of Neiyanggong on brain function}

Chan et al. (2008) applied Neiyanggong to the treatment of children with ASD [31]. Results revealed that in a portion of the cerebral cortex involved in self-control, the experimental group exhibited significantly intensified EEG activity in the cingulate gyrus $(\mathrm{t}=0.30, \mathrm{p}=0.02)$, whereas the control group did not exhibit any significant changes $(t=-0.25, p=0.81)$. This observation suggests that the intensified brain activity in the experimental group may have been induced by neural mechanisms through which Neiyanggong affects self-control in children with ASD. 


\subsubsection{Effects of Tongqiao on brain function}

Chan et al. (2009) conducted an experiment and verified that Tongqiao [32], which involves the use of Chantongganlu drops to eliminate nasal congestion, induced changes in blood flow indices of the frontal lobe, with an activity value reaching $0.64(\mathrm{t}=3.80, \mathrm{p}<0.01)$. By contrast, when Chantongganlu was replaced with normal saline solution, no significant changes were observed in either the frontal lobe cortex or anterior cingulate cortex (ACC) $(p>0.05)$. Because the frontal lobe is closely associated with intelligence and hormone balance in humans, the improvement in blood flow indices in this region may be an indicator of DMBI's effects on children with ASD. One study reported that intranasal administration of herbal medicine (i.e., Tongqiao) enhanced participants' immunologic function, as evidenced by significant reductions in circulating $\mathrm{CD} 3+\mathrm{CD} 8+$ suppressor $\mathrm{T}$ lymphocytes and cytotoxic $\mathrm{T}$ lymphocytes in blood samples [33].

\subsubsection{Effects of Shaolin Dan Tian breathing on brain function}

Research shows that Breath Therapy and others with reported benefits for a variety of health conditions[34].Dan Tian breathing (DTB) is a breathing method that is critical to the Chanwuyi health regimen. Specifically, it involves focusing on the nose and Dan Tian while exhaling and inhaling, respectively. DTB can be further divided into natural DTB and self-initiated DTB.

Chan et al. (2011) applied fMRI to determine the relationship between DTB and brain activity [35]. Preliminary results revealed that the ACC became highly active when DTB was administered. However, similar results were not observed during natural breathing. Considering that the ACC is responsible for behavioral planning and advanced behavioral regulation and that ACC dysfunction is associated with numerous brain diseases (e.g., autism and ADHD), enhancing ACC activity through DTB can be beneficial to brain function.

\subsubsection{Effects of changing dietary habits on brain function}

Chan et al. (2012) achieved remarkable results with the regulated diet prescribed in the DMBI [36]. After a 4-week dietary regimen, children with ASD exhibited substantial increases in frontal lobe and cingulate gyrus activity. These results provide evidence-based support for the clinical application of the DMBI in alleviating executive dysfunction in children with ASD.

\section{EFFECTS OF THE DMBI ON CHILDREN WITH ASD}

\subsection{IMPROVEMENT IN SELF-CONTROL}

Chan et al. (2008) employed the DMBI to enhance the self-control of a 17 year-old girl with Asperger's syndrome (a type of ASD) [37]. Before exposure to the DMBI, the girl exhibited a lack of self-control, which manifested as temper tantrums and repetitive behaviors. She lost her temper at least once per day because of trivial problems. On average, each outburst lasted for 5 to 10 minutes, with the longest being 60 minutes. She also showed a limited capability to engage in social interactions and had poor social skills. She was uninterested in socializing with people around her. Moreover, because of her lack of selfcontrol, she frequently got into arguments with others, putting further strain on social relationships. After the introduction of the 12-week DMBI program as an intervention, the girl achieved considerable improvement in her self-control abilities. The frequency of her temper tantrums decreased significantly from at least seven times per week to once or twice per week. Furthermore, the duration of outbursts also decreased from a maximum of 60 minutes to less than 5 minutes, and she was able to quickly calm down on her own. Her interest in social communication increased, and she began to actively express her care for others, demonstrating improved capability of expressing career plans and interests. Additionally, another study noted that in contrast to the muscle relaxation intervention, which only achieved slight improvements among participants in the hypofunction and severe symptoms groups, Neiyanggong achieved more comprehensive results [38].

\subsection{IMPROVEMENT IN MENTAL DEVELOPMENT}

Research shows that the DMBI can be regarded as a method to enhance cognition in children with ASD, which reduces daily self-control behavioral problems [39]. This effect is mainly reflected by differences in daily activities. Through the DMBI, one particular boy with ASD who liked to draw showed a more positive attitude and higher level of cognitive ability. Before the intervention, his drawings were simple and full of sorrow. For example, he drew weeping children beside their mothers, scenes that showcased his barren and painful inner thoughts. After participating in the DMBI, his drawings became much richer and warmer. In one drawing, he illustrated numerous rooms decorated in different styles as venues for various activities, all of which appeared to be lively and reflective of the deeper sense of joy and ease he felt. 


\subsection{REDUCTION OF REPETITIVE BEHAVIOR}

Repetitive behavior is a typical form of dysfunction noted in children with ASD, and it manifests as repetitive and impulsive actions, emotional outbursts, and impaired social communications or interactions. It is primarily induced by the impairment of executive functions, which include memory, attention, inhibition of reactions, mental flexibility, and self-governing ability. Therefore, improving the executive functioning of children with ASD can alleviate repetitive behaviors. Chan (2014) provided clinical and scientific evidence supporting the effectiveness of the DMBI in improving mood and cognitive functions. Furthermore, the DMBI has also been found to be effective in improving emotional control, behavior, memory, learning ability, immune system, and executive functioning in children with ASD.

\section{CONCLUSION AND RECOMMENDATION}

Several studies have provided statistical and neurophysiological evidence that the DMBI is beneficial to various cognitive functions, including attention, memory, self-control, and executive functioning in children with ASD. From this perspective, the DMBI has certain effects on maintaining or improving the physical and psychological health of children with ASD. For children with ASD, the DMBI is a safe, effective, and economic treatment that is worthy of greater attention.

The main methods that the DMBI uses in children with ASD are as follows: Neiyanggong, Zen meditation, regulated diet, and Tongqiao. In addition, the mechanism of action of the DMBI in children with ASD mainly involves brain function to improve physical and psychological health.

Because research concerning the effects of the DMBI on alleviating the symptoms of children with ASD is still in preliminary stages, additional time for verification and supporting evidence is required. What has been achieved so far should primarily be attributed to Professor Chan and her research team, who have spent time exploring the role of traditional culture in modern medical values. Furthermore, future research, particularly from the perspectives of other domains, will undoubtedly expand the scope and influence of this field, thereby achieving more substantial breakthroughs that will benefit patients with ASD.

\section{DATA AVAILABILITY}

The data supporting this review article are from previously reported studies, which have been cited.

\section{CONFLICTS OF INTEREST}

The authors declare that they have no conflicts of interest regarding the publication of this paper.

\section{AUTHORS' CONTRIBUTIONS}

Ming-yuan Zhao and Chen $\mathrm{Li}$, contributed equally to the study. Na Zhao acted as co-supervisors and revised the manuscript. Si-Min Huang prepared an outline, supervised, and performed the final revision of the manuscript.

\section{ACKNOWLEDGMENTS}

This work was financially supported by Project 2020 of Philosophy and Social Science Planning, Guangdong Province, China (GD20CTY13).

\section{REFERENCES}

[1] American Psychiatric Association, "Diagnostic and statistical manual of mental disorders," 5th ed. Washington DC: American Psychiatric Publishing Inc, 2013, pp. 31-86.

[2] A. Knüppel, G. K. Telléus, H. Jakobsen, M. B. Lauritsen, "Quality of life in adolescents and adults with autism spectrum disorder: Results from a nationwide Danish survey using self-reports and parental proxy-reports," Research in Developmental Disabilities, vol. 83, no. 12, pp. 247-259, Dec. 2018.

[3] W. Ying, X. Lu, R. S. Chen, "Social impairment of children with autism spectrum disorder affects parental quality of life in different ways," Psychiatry Research, vol. 266, no.8, pp. 168-174, Aug. 2018.

[4] Conner, C.M., Golt, J., Righi, G. et al. "A Comparative Study of Suicidality and Its Association with Emotion Regulation Impairment in Large ASD and US Census-Matched Samples," J Autism Dev Disord,vol.50, pp. 3545-3560 ,Jan.2020.

[5] Graetz, J. E.. "Autism grows up: Opportunities for adults with autism," Disability \& Society, vol. 25,no.1, pp.33-47,Jan.2010.

[6] Sullivan, O.A., Wang, C."Autism Spectrum Disorder Interventions in Mainland China a Systematic Review," Rev J Autism Dev Disord .no.7, pp.263277,Dec.2020.

[7] AO Yongqian,LIU Pu,WANG Tingzhao."Analysis on the Development Needs of China' s Special Education in the New Era,"Chinese Journal of Special Education,vol.237no.3,pp.15-22.2020.

[8] L. H. Chen, C. c. Lee, T.P. Ho, "Genetic overlap between ADHD and ASD in shank genes in Chinese population," European Neuropsychopharmacology, vol. 29, no.3, pp. 956-957, Mar. 2019. 
[9] Lima Antão Jennifer Yohanna Ferreira de, Oliveira Acary Souza Bulle, Almeida Barbosa Renata Thaís de, et al. "Instruments for augmentative and alternative communication for children with autism spectrum disorder: a systematic review,"Clinics, vol.73,PP.497,Nov.2018.

[10] Xu Wenxin, Yao Jiwei, Liu Wenyao. "Intervention Effect of Sensory Integration Training on the Behaviors and Quality of Life of Children with Autism".Psychiatria Danubina,Vol.31,no.3,pp.340346,Nov.2019.

[11] Richard M.FoxxPhD."Applied Behavior Analysis Treatment of Autism: The State of the ART, "Child and Adolescent Psychiatric Clinics of North America, vol.17,no.4,pp.821-834,Oct..2008.

[12] Hobson Jessica A, Tarver Laura, Beurkens Nicole, et al. "The Relation between Severity of Autism and Caregiver-Child Interaction: a Study in the Context of Relationship Development Intervention,"Journal of abnormal child Psychol, vol.44,no.4,pp.74555,Aug.2016.

[13] Gallagher S".Understanding interpersonal problems in autism:Interaction theory as analternative to theory of mind,".Philosophy, Psychiatry, \& Psychology,,vol.11,no.3,pp.199-217,Sep.2004.

[14] A. S. Chan, S. L. Sze, D. Shi, "Traditional Chinese Mind-Body Exercises Improve Self-Control Ability of an Adolescent with Asperger's Disorder," Journal of Psychology in Chinese Societies, no.9, pp. 225239, Jan. 2008.

[15] Chan, Agnes \& Sze, Sophia \& Woo, Jean \& Yu, Ruby. "A Chinese Chan-based lifestyle intervention improves memory of older adults," Frontiers in aging neuroscience, no.6.pp.50,Mar.2014.

[16] F. Winiger, "Qigong in Three Social Worlds: National Treasure, Social Signifier, or Breathing Exercise?," Health, Technology and Society, New York: Palgrave Macmillan, 2018, pp.85.

[17] Yu R, Woo J, Chan A, Sze S. "A Chinese Chanbased mind-body intervention improves psychological well-being and physical health of community-dwelling elderly: a pilot study," Clin Interv Aging,no.9,pp.727-736,Apr.2014.

[18] Green, S.A., \& Ben, S.A. "Anxiety Disorders and Sensory Over-Responsivity in Children with Autism Spectrum Disorders: Is There a Causal Relationship?" Journal of Autism and Developmental Disorders, vol.40,no.12, pp.14951504,Apr. 2010.

[19] Y. H. Kee, "Looking East for Mindfulness: A Glimpse of Practices and Research on Shaolin
Martial Arts and Related Practices to Advance Sport Psychology," Psych, vol. 1, no.1, pp. 76-91, Apr. 2019.

[20] Pedersen L, Parlar S, Kvist K, et al. Data mining the Scan Brit study of a gluten- and casein-free dietary intervention for children with autism spectrum disorders: behavioural and psychometric measures of dietary response. Nutr Neurosci, 2013, 17: 207213.

[21] L. G. Bandini, C. Curtin, M. Eliasziw, S. Phillips, L. Jay, M. Maslin, "Food selectivity in a diverse sample of young children with and without intellectual disabilities," Appetite, vol. 133, no.1, pp. 433-440, Feb. 2019.

[22] S. Siddiqi, A. Urooj, M. J. D’Souza, "Dietary Patterns and Anthropometric Measures of Indian Children with Autism Spectrum Disorder," Journal of Autism and Developmental Disorders, vol. 49, no. 4, pp. 1586-1598, Apr. 2019.

[23] Agnes S. Chan, Yvonne M. Y. Han, Sophia L. Sze, Queenie Y. Wong, Mei-chun Cheung, "A Randomized Controlled Neurophysiological Study of a Chinese Chan-Based Mind-Body Intervention in Patients with Major Depressive Disorder,"EvidenceBased Complementary and Alternative Medicine, vol. 2013, Article ID 812096, 12 pages,Dec. 2013.

[24] J. Bschevalier, "Medial temporal lobe structures and autism:a review of clinical and experimental finding," Neuropsychologia, vol. 32, no. 6 , pp. 627 648, Jun. 1994.

[25] P. K. Haist, F. Haist, F. Sedaghat, E. Courchesne, "The brain response to personally familiar faces in autism:finding of fusiform activity and beyond," Brain, vol. 127, no. 12, pp. 2703-2716, Dec. 2004.

[26] H. D. Critchley, E. Dalyem, S. Bullmore, S. C. R. Williams, T. V. Amelsvoort, D. M. Robertson, A. Rowe, M. Phillips, G. McAlonan, P. Howlin, D. G. M. Murphy, "The functional neuroanatomy of social behavior:changes in cerebral blood flow when people with autistic disorder process facial xpressions," Brain, vol. 123, no. 11, pp. 203-2212, Nov. 2000.

[27] N. Boddaert, N. Chabane, M. B. Belin, "Perception of complex sounds in autism: abnormal auditory cortical processing in children," American Journal of Psychiatry, vol. 161, no. 11, pp. 2117-2120, Nov. 2004.

[28] E. L. Hill, "Executive dysfunction in autism," Trends in Cognitive Sciences, vol. 8, no. 1, pp. 26-32, Jan. 2004. 
[29] K. Francis, A. Dougali, K. Sideri, C. Kroupis, V. Vasdekis, K. Dima, A. Douzenis, "Brain - derived neurotrophic factor (BDNF) in children with ASD and their parents: a 3 - year follow - up," Acta Psychiatrica Scandinavica, vol.137, no. 5, pp. 433441, May. 2018.

[30] N.Li, J. Yang, J. Zhang, "Correlation of Gut Microbiome Between ASD Children and Mothers and Potential Biomarkers for Risk Assessment.Genomics," Proteomics \& Bioinformatics, vol. 17, no. 1, pp .26-38, Feb. 2019.

[31] A. S. Chan, S. L. Sze, D. Shi, "Traditional Chinese Mind-Body Exercises Improve Self-Control Ability of an Adolescent with Asperger's Disorder," Journal of Psychology in Chinese Societies, no.9, pp.225239, Jan. 2008.

[32] A. S. Chan, S. L. SZE, M. C. Cheung, M. K. Lam, D. Shi, "Dejian mind-body intervention improves the functioning of a patient with chronic epilepsy: a case report," Cases journal, no.4. pp. 1-7, Nov. 2009.

[33] A.S. Chan, Y. M. Y. Han, S. L. Sze, E. M. Lau, "Neuroenhancement of Memory for Children with Autism by a Mind-Body Exercise," Front Psychology.6:1893.

DOI:10.3389/fpsyg.2015.01893.

[34] Mehling, W. E., Wrubel, J., Daubenmier, J. J., Price, C. J., Kerr, C. E., Silow, T., Gopisetty, V., \& Stewart, A. L. (2011). Body Awareness: a phenomenological inquiry into the common ground of mind-body therapies. Philosophy, ethics, and humanities in medicine: PEHM, 6, 6. https://doi.org/10.1186/1747-5341-6-6

[35] A. S. Chan, M. C. Cheung, S. L. Sze, W. W. M. Leung, D. Shi, "Shaolin Dan Tian Breathing Fosters Relaxed and Attentive Mind: A Randomized Controlled Neuro-Electrophysiological Study," Evidence-Based Complementary and Alternative Medicine, DOI: org/10.1155/2011/180704.

[36] A. S. Chan, S. L. Sze, Y. M. Y. Han, M. C. Cheung, "A Chan Dietary Intervention Enhances Executive Functions and Anterior Cingulate Activity in Autism Spectrum Disorders: A Randomized Controlled Trial," Evidence-Based Complementary and Alternative Medicine, DOI: org/10.1155/2012/262136.

[37] A. S. Chan, S. L. Sze, D. Shi, "Traditional Chinese Mind-Body Exercises Improve Self-Control Ability of an Adolescent with Asperger's Disorder," Journal of Psychology in Chinese Societies, no. 9, pp. 225239, Jan. 2008.
[38] Lau, Mei Ting Eliza, "Treatment Effects of Chanbased Mind Body Exercise and Progressive Muscle Relaxation on Impulse Control and Cognitive Flexibility of Children with Autism Spectrum Disorder: A Randomized Controlled Trial," M.S.thesis, Dept. Psychology, The Chinese University of Hong Kong, Hong Kong, China, 2017.

[39] A. S. Chan, S. L. Sze, N. Y. Siu, E. M. La, M. C. Cheung, "A Chinese Mind-Body Exercise Improves Self-Control of Children with Autism: A Randomized Controlled Trial," PLoS One. DOI: org/10.1371/journal.pone. 0068184. 